\title{
Erratum to: Proteomic Analysis of Somatic Embryogenesis in Cyclamen persicum Mill
}

\author{
Fuhua Bian • Caixia Zheng • Funing Qu • \\ Xueqin Gong • Cuirong You
}

Published online: 10 February 2010

(C) Springer-Verlag 2010

Erratum to: Plant Mol Biol Rep, 28(1):22-31 (2010)

DOI 10.1007/s11105-009-0104-5

The corresponding author of this article is Funing Qu instead of Fuhua Bian as previously indicated. The metadata should be read as:

F. Bian • F. Qu $(\bowtie) \bullet$ X. Gong $\bullet$ C. You

College of Chemistry and Biology Technology,

Yantai University, Yantai 264005,

People's Republic of China

e-mail: qfnzp@ytu.edu.cn

C. Zheng

College of Biological Sciences and Biotechnology,

Beijing Forestry University

Beijing 100083, People's Republic of China

The online version of the original article can be found at http://dx.doi. org/10.1007/s11105-009-0104-5.

F. Bian $\cdot$ F. Qu $(\bowtie) \cdot X$. Gong $\cdot$ C. You

College of Chemistry and Biology Technology, Yantai University,

Yantai 264005, People's Republic of China

e-mail: qfnzp@ytu.edu.cn

C. Zheng

College of Biological Sciences and Biotechnology,

Beijing Forestry University,

Beijing 100083, People's Republic of China 